\title{
Development and validation of a predictive nomogram for the risk of recurrence in patients with cystitis glandularis
}

\author{
Jiao Hu ${ }^{1 \#}$, Chao Li1" ${ }^{1 \#}$ Xi Guo ${ }^{2}$, Huihui Zhang ${ }^{3}$, Huihuang Li $^{1}$, Dongxu Qiu ${ }^{1}$, Guanghui Gong ${ }^{4}$, Peihua Liu ${ }^{1}$, \\ Wenbiao Ren ${ }^{1}$, Jinbo Chen ${ }^{1}$, Xiongbing $\mathrm{Zu}^{1}$ \\ ${ }^{1}$ Department of Urology, Xiangya Hospital, Central South University, Changsha 410008, China; ${ }^{2}$ Department of Urology, Hunan Provincial People's \\ Hospital, Changsha 410005, China; ${ }^{3}$ Department of Urology, the First Affiliated Hospital of the University of South China, Hengyang 421001, \\ China; ${ }^{4}$ Department of Pathology, Xiangya Hospital, Central South University, Changsha 410008, China \\ Contributions: (I) Conception and design: Hu, C Li, X Zu, J Chen; (II) Administrative support: J Chen, X Zu; (III) Provision of study materials or \\ patients: J Hu, C Li, H Li, X Guo, H Zhang, G Gong, W Ren; (IV) Collection and assembly of data: J Hu, C Li, P Liu, D Qiu, W Ren; (V) Data \\ analysis and interpretation: J Hu, C Li, P Liu; (VI) Manuscript writing: All authors; (VII) Final approval of manuscript: All authors. \\ \#These authors contributed equally to this work. \\ Correspondence to: Xiongbing Zu; Jinbo Chen. Department of Urology, Xiangya Hospital, Central South University, Changsha 410008, China. \\ Email: zuxbxyyy@126.com; chenjinbo1989@yahoo.com.
}

Background: Most patients with cystitis glandularis (CG) suffer from recurrence after primary treatment. Therefore, we performed this multicenter study to clarify the recurrent risk factors and constructed a predictive nomogram for the risk of recurrence. Also, we try to investigate the correlation between CG and bladder cancer.

Methods: Consecutive patients with pathologically confirmed CG were divided into training and validation sets. Clinicopathological characters were collected from electronic medical records. Uni- and multivariate logistic regression analyses were used to identify independent risk factors of CG recurrence in the training set. The predictive nomogram was developed by incorporating these independent factors and histological subtype. The performance of the nomogram was assessed and validated with respects to its calibration, discrimination, and clinical usefulness. The risk of developing subsequent bladder cancer was analyzed from the follow-up data.

Results: Ultimately, 278 eligible patients were included and were allocated to a training set $(\mathrm{n}=190)$ and a validation set ( $\mathrm{n}=88)$. Of them, $165(59.35 \%)$ patients experienced CG recurrence, and none showed evidence of subsequent bladder carcinoma during a median (IQR) follow-up time of 27 months (14-57 months). Results of multivariate analysis showed that urinary infections, long-term indwelling catheter usage, urinary calculus, squamous metaplasia, and atypical hyperplasia were independent risk factors of CG recurrence. The C-index (95\% CI) of the nomogram was $0.76(0.69-0.83)$ in the training set and $0.72(0.61-0.83)$ in the validation set. A decision curve analysis (DCA) demonstrated that this predictive nomogram was clinically useful.

Conclusions: We developed and validated a nomogram to predict the individualized risk of CG recurrence. Also, we demonstrated that neither intestinal nor typical CG increased the consequent risk of bladder cancer during the follow-up period.

Keywords: Cystitis glandularis (CG); nomogram; recurrence

Submitted Nov 27, 2019. Accepted for publication Jan 30, 2020.

doi: 10.21037/atm.2020.02.102

View this article at: http://dx.doi.org/10.21037/atm.2020.02.102 


\section{Introduction}

Cystitis glandularis (CG) was regarded as a rare disease with an incidence of approximately $1 \%$ (1). Now, it is more common with increasing inflammatory stimuli and the development of urological endoscopic technology (2). Its manifestations are non-specific, and include bladder irritation and hematuria (3). Pathologically, it is an abnormal proliferative disorder of the bladder's transitional epithelium characterized by von Brunn's nests (4). There are two pathological subtypes, typical and intestinal, that differ morphologically and biologically $(5,6)$.

The etiologies of CG remain unclear. A long-standing stimulus such as a urinary infection, urine retention, and bladder calculus play an essential role in the development of CG by inducing transitional bladder epithelium to glandular epithelium (6-9). Also, we recently revealed that IncRNA UCA1 promoted the development of CG by increasing the cell viability, proliferation, and migratory potential of CG cells (10). Transurethral resection and conservative treatments, which include antibiotic therapy and symptomatic treatment, are the most common treatment options for CG $(11,12)$. Many patients suffer from CG recurrence after receiving primary treatments $(13,14)$. However, no previous study has investigated the risk factors of CG recurrence. Moreover, the definition of CG recurrence in previous studies only included the pathological recurrence, as confirmed by cystoscopy and biopsy. In fact, symptomatic recurrence may be the leading reason for retreatment and reduction of life quality. Here, we redefined the CG recurrence by integrating symptomatic and pathological recurrence and systematically analyzed the recurrent risk factors.

Several previous case reports showed that patients with CG developed bladder adenocarcinoma, which prompted the consideration of CG as a precancerous lesion $(15,16)$. Recently, some studies illustrated that there was no direct association between typical CG and bladder carcinoma (17-19). But debates still persist regarding the malignant potential of intestinal CG. Intestinal CG resembles bladder cancer in many aspects. Immunologically, the expression of cyclooxygenase-2 and B-cell lymphoma-2, two potential tumor initiators and promoters, were significantly higher in intestinal CG compared with typical CG and normal bladder tissues (20). Clinically, several studies observed emerging bladder cancer in patients with intestinal CG during the follow-up period $(3,21)$. However, other studies produced findings that did not support intestinal CG as a precancerous disease $(18,19)$.

Given the rarity of CG, most previous studies were small sample-sized and carried out at a single institution. This was a multi-center study to reveal the risk factors of CG recurrence and explore the correlation between CG and bladder cancer.

\section{Methods}

Ethical approval was obtained from three general tertiary hospitals, including Xiangya Hospital, Hunan Provincial People's Hospital, and the First Affiliated Hospital of the University of South China. Written informed consent was obtained from all patients. The entire cohort of this study was identified following an evaluation of the medical records system, from January 2010 to January 2018. We identified patients with pathologically confirmed CG. The patient inclusion flow diagram is shown in Figure 1. The entire cohort was randomly allocated to training and validation sets with a ratio of 7:3. All patients received regular followup examinations, including serial cystoscopies, bladder biopsies, ultrasound, and urine cytology.

\section{Data collection}

The clinical characteristics, including age, sex, smoking history, drinking history, and main symptoms, were obtained from the electronic medical record system. Also, urologic history details, including history of urinary infection, urinary calculus, bladder outlet obstruction, long-term indwelling catheters, and upper urinary tract obstructions, were collected. The pathological features, including squamous metaplasia, histological subtype, and atypical hyperplasia, were re-evaluated by two independent pathologists who were blind to the clinical data.

\section{Study outcomes}

The primary outcome was to identify the risk factors of CG recurrence and construct a predictive nomogram for individualized risk of recurrence. In this study, CG recurrence was defined according to pathological and symptomatic recurrence. Pathological recurrence was defined as follows: new CG lesions reappeared after the primary CG lesions were completely resected. Symptomatic recurrence was defined as: new obvious symptoms that reappeared during the follow-up period when the primary symptoms had disappeared completely after receiving 


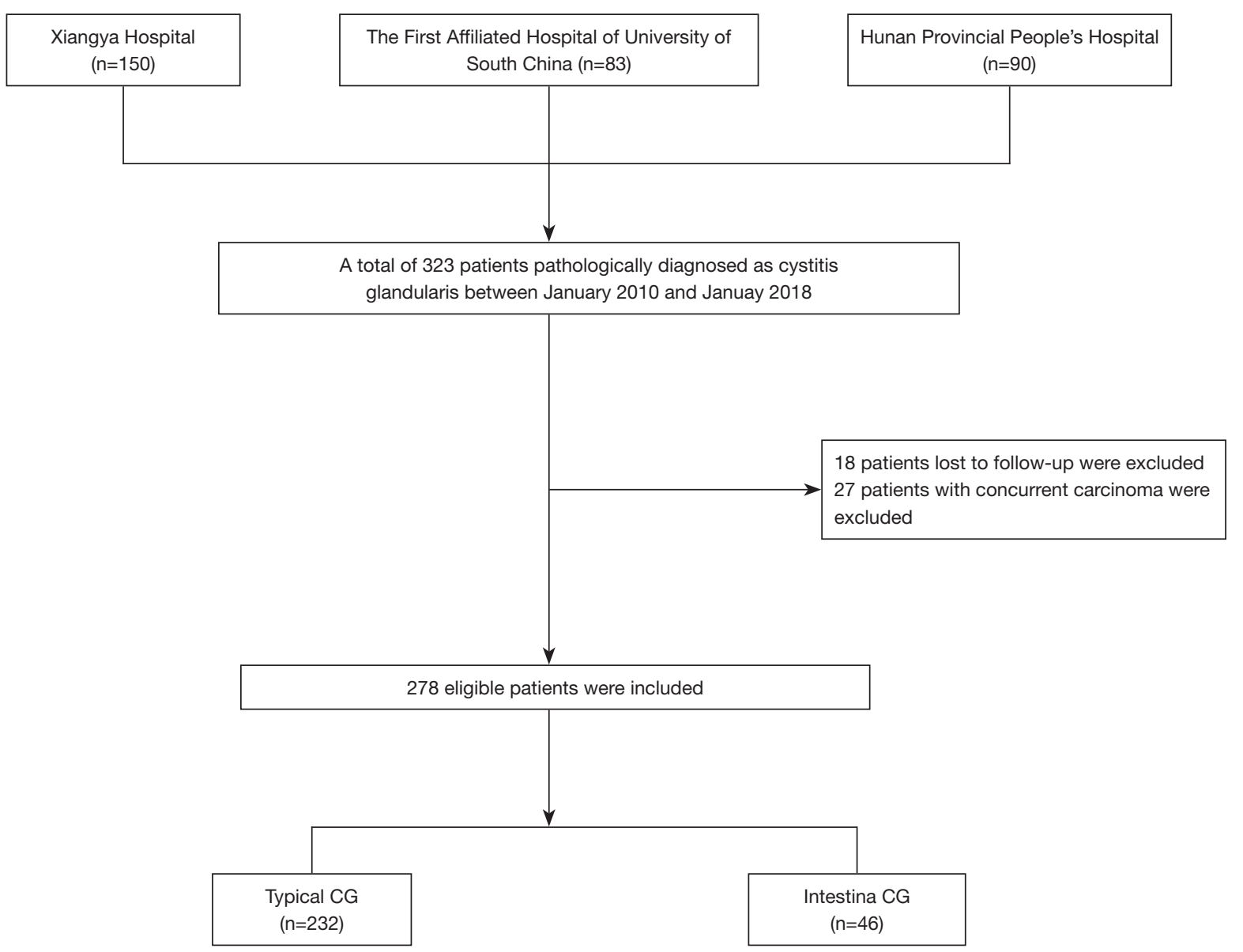

Figure 1 Flow diagram of included patients.

surgery or conservative treatments. These new symptoms could not be explained by other urinary diseases. Secondary outcomes were to explore the correlation between CG and bladder cancer and compare the difference between intestinal and typical CG.

\section{Development and validation of a nomogram to predict the risk of recurrence}

To develop a predictive nomogram, we used multivariable logistic regression analysis to identify the risk factors that were significantly correlated with recurrence and combined them to construct an inclusive model using the training set. Then we determined the predictive accuracy and discrimination ability of the nomogram in the training set using the C-index and calibration plot, respectively. A bootstrapping validation (1,000 bootstrap resamples) was used to calculate a corrected C-index. Also, a visualized receiver operator characteristic (ROC) curve was plotted to assess the predictive accuracy. The calibration curve was plotted to assess the goodness-of-fit of the nomogram by using the Hosmer-Lemeshow test. Validation of the predictive nomogram was performed in the validation set. A predictive score for all patients in the validation set was calculated using the predictive nomogram constructed in the training set. Then a logistic regression in the validation set was performed by using the predictive score as a factor. Finally, the C-index, ROC curve, and calibration curve were derived based on the regression analysis. To estimate the clinical usefulness of the nomogram, we performed a decision curve analysis (DCA) to calculate the net benefits for a range of threshold probabilities in the training set.

\section{Statistical analysis}

Statistical analysis was performed using SPSS (version 22.0, 
IBM) and R statistical software (version 3.3.3, https://www. r-project.org). Uni- and multivariate logistic regression analyses were used to identify independent risk factors for CG recurrence. Nomogram construction and calibration plots were performed using the "rms" package. The ROC curves were plotted using the "pROC" package. DCA was performed using the "dca.R." package. Differences between intestinal and typical CG were analyzed using the chi-square test. Two-sided $\mathrm{P}$ values less than 0.05 were considered statistically significant.

\section{Results}

\section{General characteristics}

Ultimately, 278 eligible patients with CG (mean age, $50.64 \pm 13.06$ years) were included and were randomly allocated to a training set $(\mathrm{n}=190)$ and a validation set $(\mathrm{n}=88)$. There was no difference in the demographic characteristics between the two sets. The patients' demographic characteristics are presented in Table 1. In the current study, frequent micturition $(61.87 \%)$, urinary urgency $(43.17 \%)$, urethral pain (41\%), hematuria (34\%), nocturia (14\%), dysuria (12\%), bladder pain $(11 \%)$, and incomplete emptying (7\%) were the most common symptoms of patients with CG. Overall, 165 (59.35\%) patients experienced CG recurrence. None died or showed evidence of subsequent bladder carcinoma during a median (IQR) follow-up time of 27 months (14-57 months).

\section{Risk factors of CG recurrence}

There were 111 patients that experienced recurrence in the training set. Results of the univariable analysis indicated that sex, urinary infection, long-term indwelling catheter usage, urinary calculus, atypical hyperplasia, and histological subtype were related to CG recurrence (Table 2). Then, we performed further multivariable logistic analysis using the factors with a $\mathrm{P}$ value of less than 0.10 in the univariable analysis. Results of multivariate analysis showed that urinary infection (OR, 5.19; 95\% CI, 1.32-20.44), long-term indwelling catheter usage (OR, 5.98; 95\% CI, 1.52-23.59), urinary calculus (OR, 5.38; 95\% CI, 1.66-17.51), squamous metaplasia (OR, 5.11; 95\% CI, 1.23-21.24), and atypical hyperplasia (OR, 5.10; 95\% CI, 1.72-15.10) were independent risk factors of $\mathrm{CG}$ recurrence.

\section{Development of predictive nomograms for $C G$ recurrence}

The first predictive nomogram that incorporated the above independent risk factors was developed and is depicted in Figure $2 A$. Histological subtype was no longer a significant risk factor in multivariable analysis; however, given its critical clinical value, we constructed a second nomogram by incorporating the histological subtype based on the first nomogram (Figure 2B). Then we assessed the predictive accuracy of two nomograms with respects to their ROC curves, Net Reclassification Index (NRI), and integrated discrimination improvement (IDI) (Figure 3). We found that the predictive accuracy improved after introduced the histological subtype to the first nomogram (NRI (continuous) was $0.31, \mathrm{P}<0.001$; the AUC improved to 0.76 from 0.72; IDI was $0.02, \mathrm{P}=0.023)$. Therefore, we selected the second nomogram as the final model.

\section{Performance of the final nomogram in the training and validation set}

In the training set, the C-index (95\% CI) of the final nomogram was $0.76(0.69-0.83)$. The ROC curve was plotted to evaluate the discrimination ability and is shown in Figure $4 A$. The calibration plot showed satisfactory agreement between the predicted risk of recurrence and actual observation (Figure $4 B$ ). In the validation set, the C-index (95\% CI) of the nomogram was $0.72(0.61-0.83)$. The ROC curve was shown in Figure 4C. Satisfactory calibration was also observed for the probability of CG recurrence in the validation cohort (Figure 4D). The decision curve analysis was presented in Figure 5. This indicated that, if the threshold probability was more than $15 \%$, using the nomogram to predict CG recurrence added more benefit than either the treat-all-patients scheme or the treat-none scheme.

\section{Differences in clinicopathological features between typical and intestinal CG}

In the entire set, $46(16.55 \%)$ patients were diagnosed with intestinal CG. Pathologically, intestinal CG featured intestinal-like structures and plentiful mucin-secreting goblet cells. Typical CG was characterized by nests of columnar cells within the lamina propria of the bladder that form glandular structures. Representative images 
Table 1 Characteristics of patients in the training and validation sets

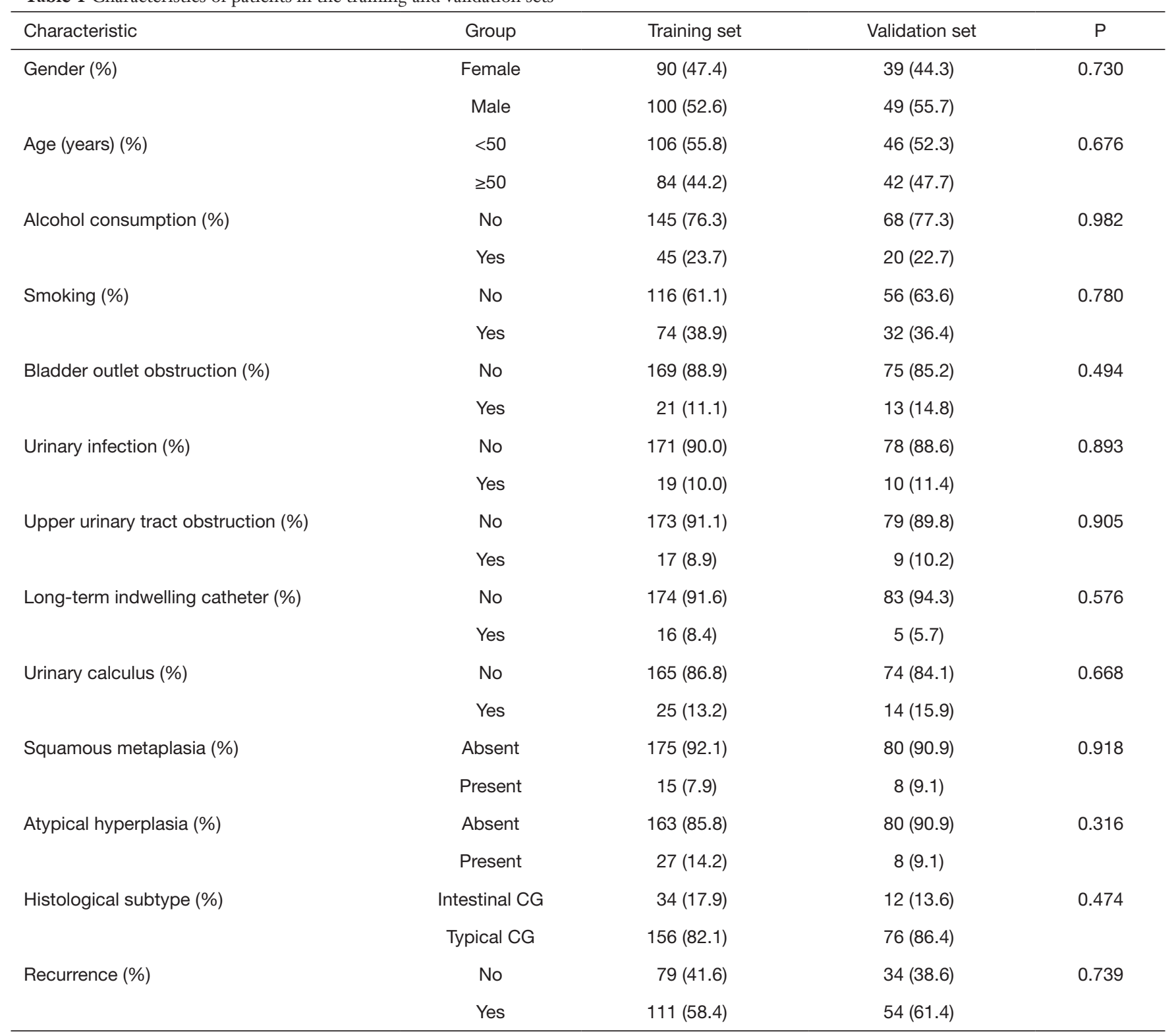

CG, cystitis glandularis.

of two subtypes were shown in Figure 6. Compared with the typical CG group, the proportion of males in the intestinal group was higher, the patients were younger, and the incidence of urinary infection was higher. Also, the recurrence rate of the intestinal group was significantly higher than that of the typical group ( $82.6 \%$ vs. $54.7 \%$; $\mathrm{P}<0.001$ ) (Table 3). During the follow-up period, both intestinal and typical group showed no evidence of consequent bladder cancer.

\section{Discussion}

To the best of our knowledge, this is the first multicenter study with a large sample size to explore the recurrent risk factors and the malignant potential of CG. We found that the recurrence rate of $\mathrm{CG}$ reached $59.35 \%$, which was far more than that of previous studies $(13,14)$. Such a high recurrence rate may be partly due to the introduction of the concept of symptomatic recurrence. There were two reasons to introduce this concept. First, pathological recurrence was 
Table 2 Risk factors of CG recurrence in the training set

\begin{tabular}{|c|c|c|c|c|c|c|}
\hline Characteristic & \multicolumn{3}{|c|}{ Univariable analysis } & \multicolumn{3}{|c|}{ Multivariable analysis } \\
\hline Gender (male vs. female) & 2.12 & $1.18-3.82$ & 0.012 & 1.58 & $0.78-3.21$ & 0.203 \\
\hline Age (years) ( $\geq 50$ vs. $<50)$ & 0.91 & $0.51-1.63$ & 0.750 & - & - & - \\
\hline Smoking (yes vs. no) & 0.85 & $0.47-1.54$ & 0.594 & - & - & - \\
\hline Bladder outlet obstruction (yes vs. no) & 0.85 & $0.33-2.16$ & 0.731 & - & - & - \\
\hline Urinary infection (yes vs. no) & 4.27 & $1.20-15.19$ & 0.025 & 5.19 & $1.32-20.44$ & 0.019 \\
\hline Upper urinary tract obstruction (yes vs. no) & 0.75 & $0.26-2.11$ & 0.583 & - & - & - \\
\hline Long-term indwelling catheter (yes vs. no) & 3.36 & $0.92-12.22$ & 0.066 & 5.98 & $1.52-23.59$ & 0.011 \\
\hline Atypical hyperplasia (present vs. absent) & 3.66 & $1.32-10.13$ & 0.013 & 5.10 & $1.72-15.10$ & 0.003 \\
\hline
\end{tabular}

$P$ value, in the logistic regression analyses; variables found to be statistically significant or nearly significant $(P<0.1)$ in the univariate analysis were entered into the nest multivariate logistic regression analysis. CG, cystitis glandularis.

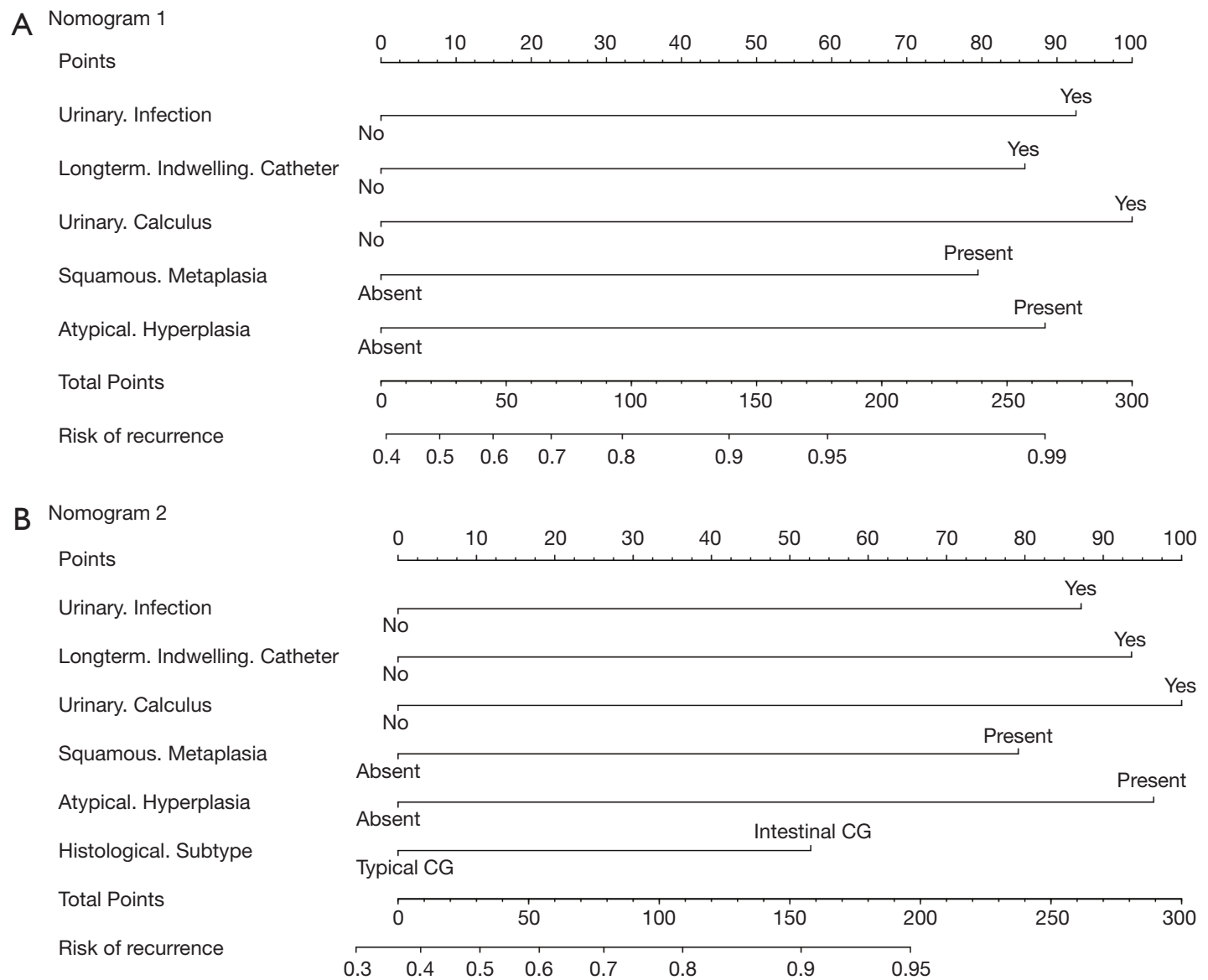

Figure 2 Two nomograms and their comparisons. (A) Nomogram 1; (B) Nomogram 2. 
A

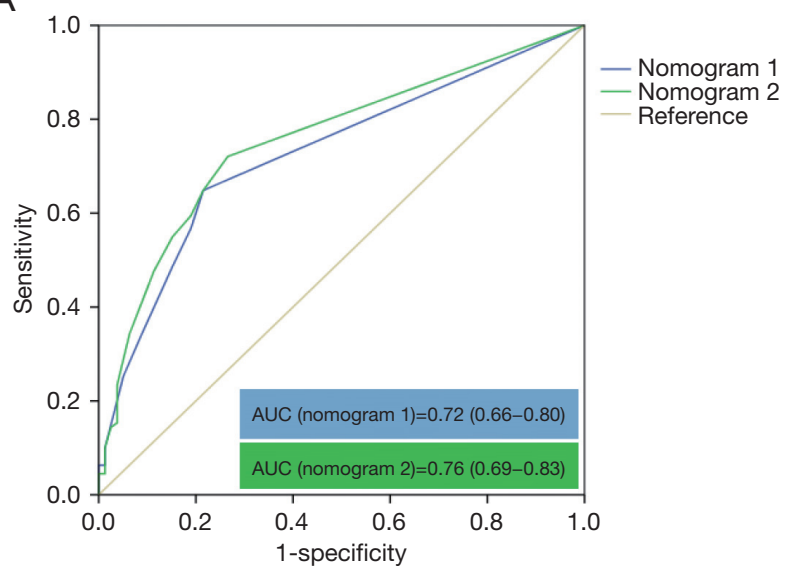

B

Nomogram 2 compared with nomogram 1

\begin{tabular}{|c|c|c|}
\hline Index & Value $(95 \% \mathrm{Cl})$ & $\mathrm{P}$ \\
\hline NRI (Categorical) & $0.02(-0.05-0.09)$ & 0.538 \\
\hline NRI (Continuous) & $0.31(0.11-0.51)$ & $<0.001$ \\
\hline IDI & $0.02(0.01-0.04)$ & 0.023 \\
\hline
\end{tabular}

Figure 3 ROC curves of two nomograms and their comparisons. (A) Discrimination ability of two nomograms measured by ROC curves; (B) the NRI and IDI of two nomograms. ROC, receiver operator characteristic; NRI, Net Reclassification Index; IDI, integrated discrimination improvement.

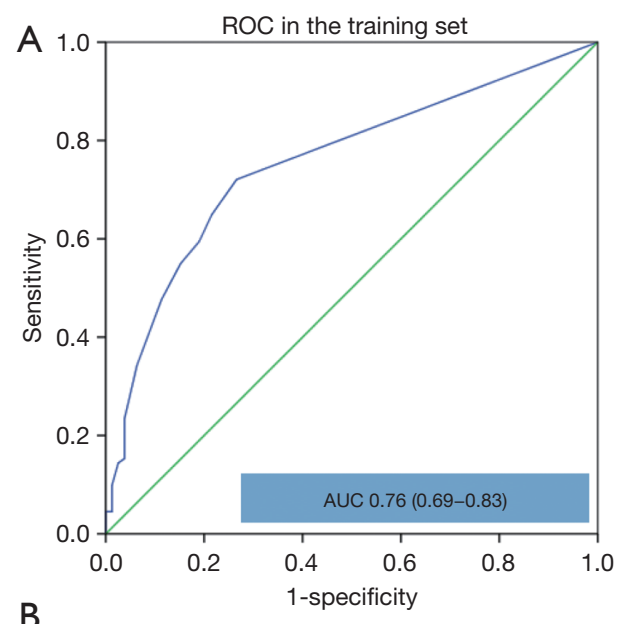

B

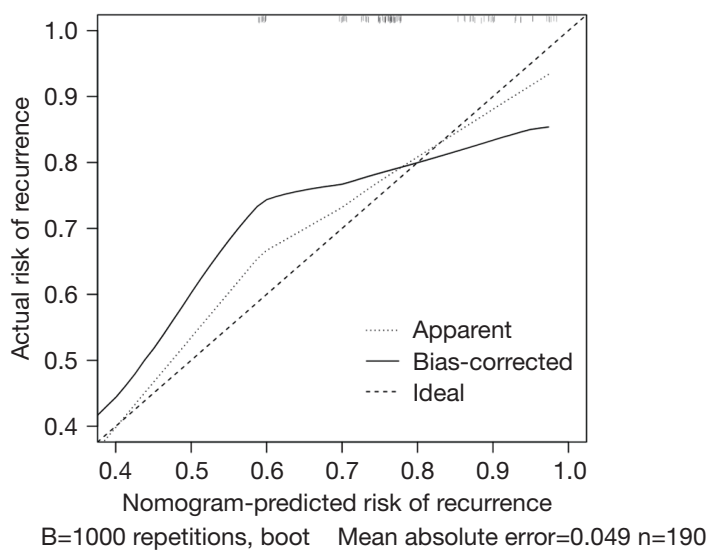

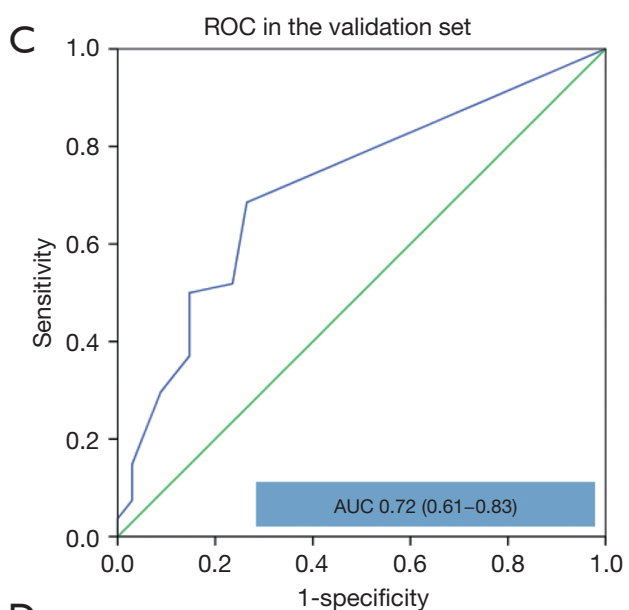

D

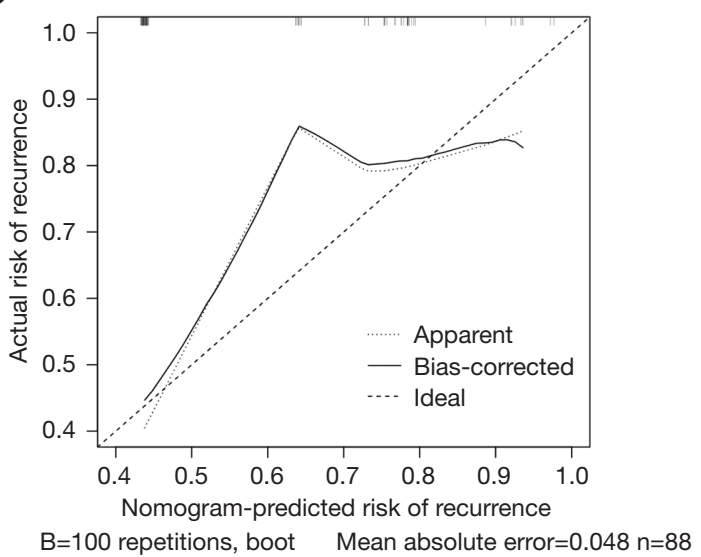

Figure 4 Performance of the nomogram 2 in the training and validation set. (A) ROC curve in the training set; (B) calibration measured by Hosmer-Lemeshow test in the training set; (C) ROC curve in the validation set; (D) calibration curve in the validation set. The calibration curve was plotted to evaluate the agreement between the predicted risks of CG recurrence and actual risk of recurrence. The y-axis represented the actual risk of recurrence. The $\mathrm{x}$-axis represented the predicted risk of recurrence. The diagonal dotted line represented a perfect prediction by an ideal model. ROC, receiver operator characteristic. ROC, receiver operator characteristic. 


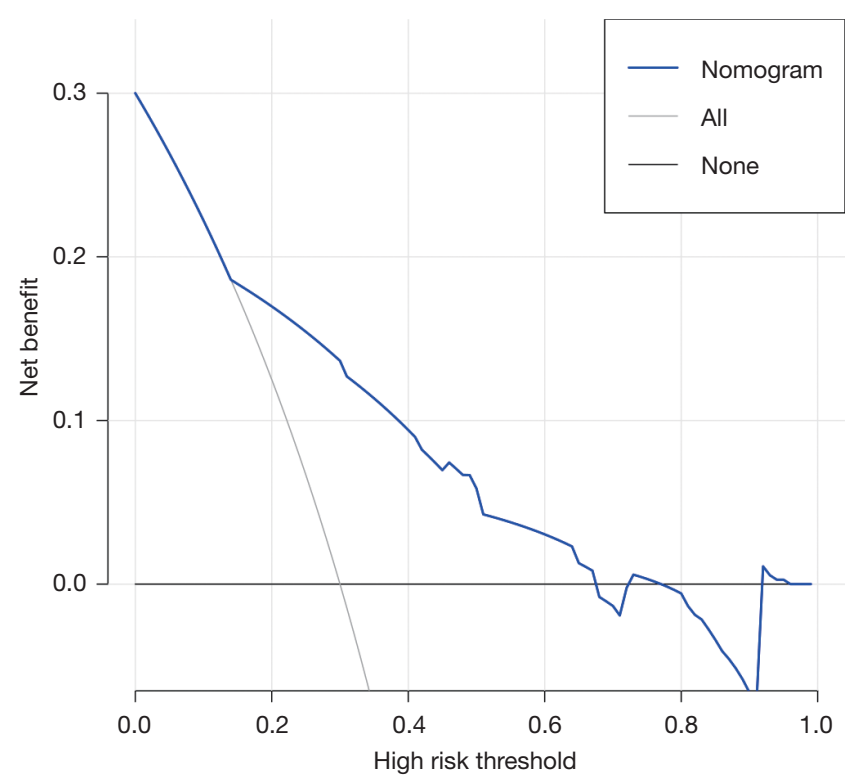

Figure 5 Clinical decision curve in the training set. The y-axis represented the net benefit. The blue line represented the nomogram. The net benefit was calculated by subtracting the proportion of all patients who were false positive from the proportion who are truly positive, weighting by the relative harm of giving up treatment compared with the negative consequences of an overtreatment. The $\mathrm{x}$-axis represented the threshold probability.

only suitable for patients who received surgery. Therefore, we cannot assess the pathological recurrence in patients who received conservative treatments. Moreover, some patients who were cured by surgery may have obvious symptoms reappear that cannot be explained by other urinary diseases, and there was no evidence of pathological recurrence in cystoscopy and biopsy. Hence, introducing the definition of symptomatic recurrence may enable us to comprehensively assess the CG recurrence.

In the present study, we revealed that the urinary infection, long-term indwelling catheter usage, urinary calculus, squamous metaplasia, and atypical hyperplasia were independent risk factors for CG recurrence. Then, we developed and validated a nomogram by integrating these independent factors and histological subtype. Although this nomogram can guide decision-making and enable us to predict the individualized risk of CG recurrence (to some extent), the overall performance was barely satisfactory with a C-index of 0.76 . There were several possible reasons for this barely satisfactory accuracy. First, this was a retrospective study, which could cause bias in the collection and processing of data. Second, the potential predictors included in the nomogram were all qualitative. Compared with quantitative predictors, qualitative predictors are more subjective. Therefore, we should improve the performance of the nomogram by introducing some quantitative disease markers in the future. Third, the recurrence in this study included symptomatic and pathological recurrence. However, the concept of systematic recurrence of CG was first proposed here, and it has not been validated in external studies. Therefore, further prospective study was warranted to validate the effectiveness of our nomogram and verify the clinical application of the concept of systematic recurrence. Based on the clinical decision curve analysis, we found that this nomogram added more benefit than either the treatall-patients scheme or the treat-none scheme when the threshold probability was more than $15 \%$.

There is no known correlation between typical CG and bladder cancer (17-19). The current debate centered around the question of whether intestinal CG was a premalignant lesion. Some investigators revealed several similarities between intestinal CG and bladder cancer. Li et al. found that intestinal CG highly expressed some tumorassociated molecules, such as cyclooxygenase- 2 and B-cell lymphoma-2 (20). Xin et al. retrospectively analyzed the follow-up data of 86 patients with intestinal CG (3). They found that one patient developed new bladder cancer. A common limitation in these studies was the small number of participants and events. By contrast, some studies with longer follow-up times demonstrated that intestinal CG was a benign lesion. Corica et al. investigated 53 patients with intestinal CG and found that none developed bladder carcinoma after a median follow-up of 13 years (18). Smith et al. analyzed 117 patients with CG and demonstrated that intestinal CG lesions could be identified in benign bladder tissues or in conjunction with bladder cancer tissues. They found no evidence that intestinal CG increased the future risk of bladder cancer (19). Yi et al. (17) evaluated the association between CG and bladder cancer in 166 patients and revealed that none developed bladder cancer during a median follow-up of 2.67 years. Moreover, repeated cystoscopies in a short time after primary treatment were not recommended in these studies. Similarly, no patients in our study developed bladder carcinoma during a median follow-up of 27 months (14-57 months). To conclude, we are more inclined to support the view that intestinal is a benign lesion, and it will not increase the future risk of bladder cancer.

In addition to the morphologic differences between intestinal and typical CG, our findings revealed their 
$A_{1}$

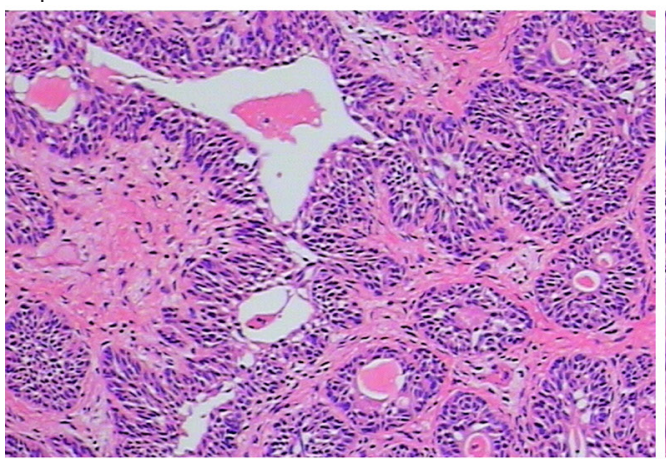

$B_{1}$

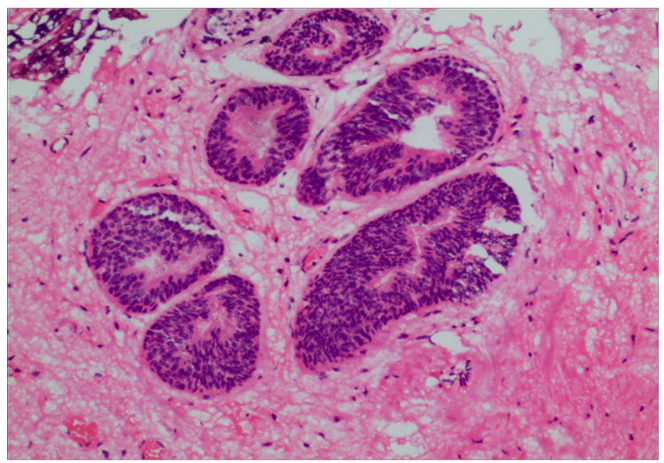

$\mathrm{C}_{1}$

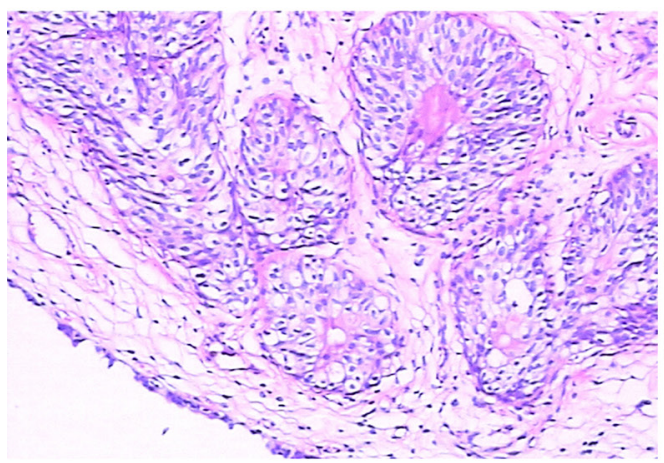

$D_{1}$

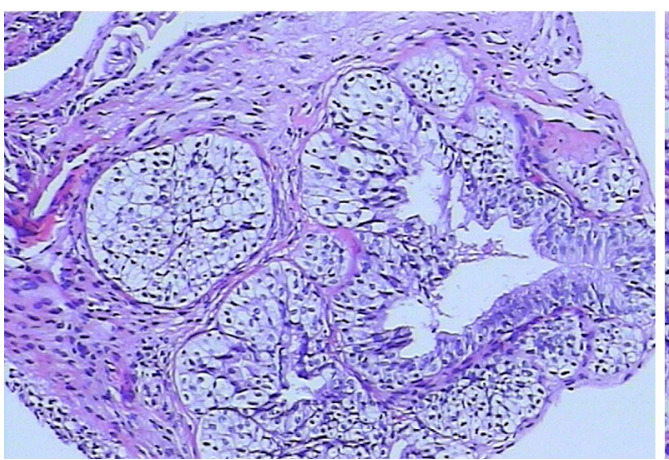

$\mathrm{A}_{2}$

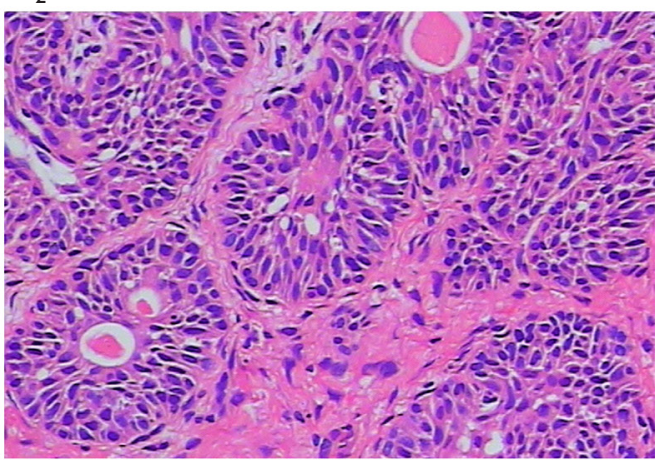

$\mathrm{B}_{2}$

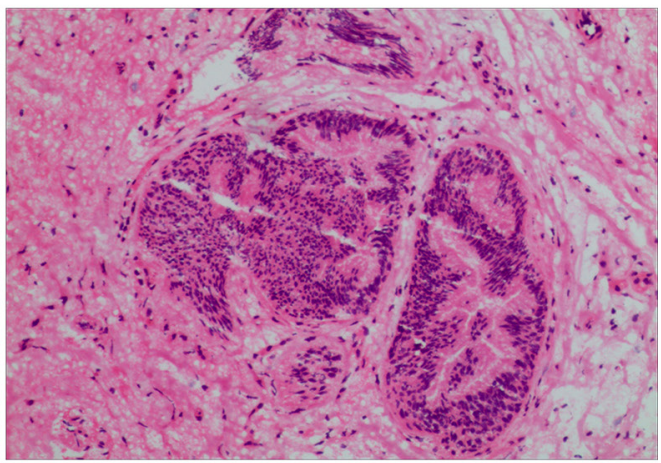

$\mathrm{C}_{2}$

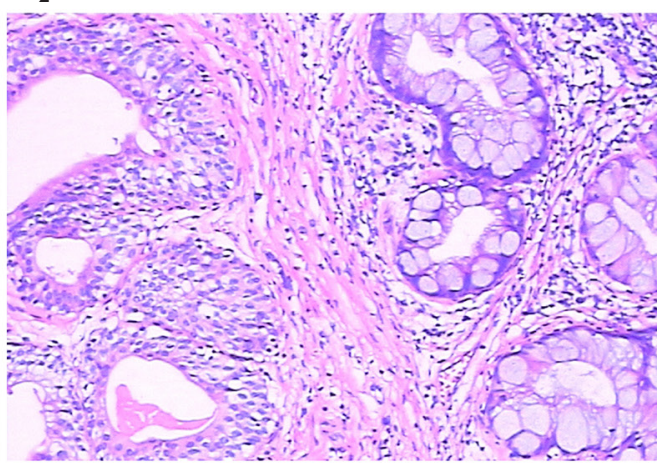

$D_{2}$

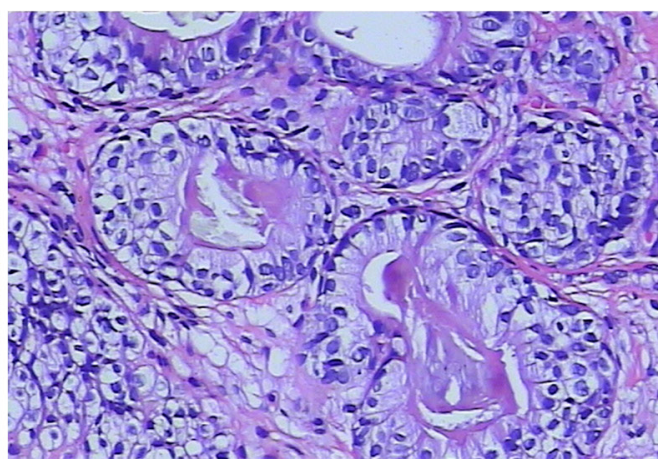

Figure 6 Pathological features of the intestinal and typical CG in HE stained sections. (A,B) typical CG; (C,D) intestinal CG; The subscript 1 means low-power field $(4 \times 10)$. The subscript 2 means a high-power field $(10 \times 10)$. CG, cystitis glandularis. 
Table 3 Differences in clinicopathological characteristics between typical and intestinal CG in the entire set

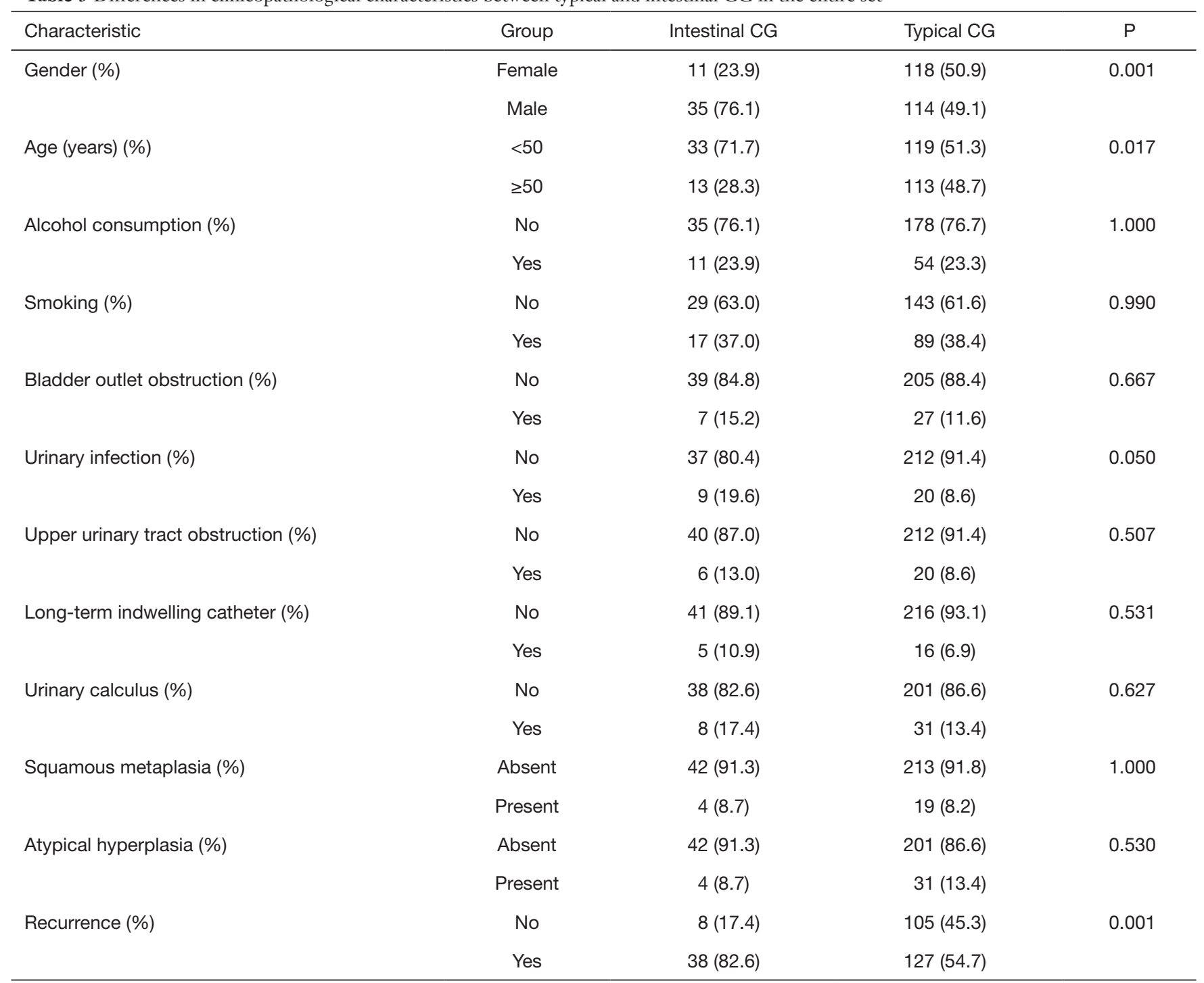

CG, cystitis glandularis.

biological behaviors were also different. The intestinal CG was more common in younger patients, males, and those with histories of urinary infections. This was similar to the findings reported by Xin et al. (3). Also, the recurrence rate of intestinal CG was significantly higher than that of typical CG $(82.6 \%$ vs. $54.7 \%$, respectively). But the underlying mechanism for these different biological behaviors is poorly understood. We hypothesized that this may be related to the obvious differences in the protein expression patterns between two CG types. Sung et al. confirmed that the expression of CDx2 and CK20, which played critical roles in the differentiation and maintenance of intestinal epithelium, were significantly higher in intestinal CG than that in typical CG (6). In contrast, CK7, an intermediate weight cytokeratin, was rarely expressed in intestinal CG, but positively expressed in all cases of typical CG. Velickovic et al. found that MUC2 and MUC5AC were specifically expressed in intestinal CG, while MUC1 and CD10 were specifically expressed in typical CG (5). Given the differences in morphology, biological behaviors, and protein expression profile between intestinal and typical CG, more targeted and specific treatment options are urgently needed.

There are several drawbacks to this study. First, the level of evidence was limited by the retrospective nature of 
this study. Second, the definition of systematic recurrence was first proposed in this study. Therefore, further studies are required to confirm this concept. Third, we have not explored the molecular mechanism of CG recurrence, but this is our future research direction. Fourth, the prediction effect of our nomogram was barely satisfactory. Therefore, its clinical value requires further verification.

\section{Conclusions}

We developed a nomogram to predict the individualized risk of CG recurrence by integrating urinary infection, long-term indwelling catheter, urinary calculus, squamous metaplasia, atypical hyperplasia, and intestinal subtype. This nomogram was well fitted and achieved high accuracy. Also, we demonstrated that neither intestinal nor typical CG would increase the consequent risk of bladder cancer. The intestinal CG significantly differed from typical CG in biological behaviors.

\section{Acknowledgments}

Funding: This work was supported by the National Natural Science Foundation of China (81572523, 81700665, 81873626, 81902592), the Hunan Province Funds for Distinguished Young Scientists of China (2016JJ1026), Hunan Province Key R\&D Program (2019SK2202) and Xiangya Hospital Youth Fund (2018Q09).

\section{Footnote}

Conflicts of Interest: JC serves as an unpaid section editor of Annals of Translational Medicine from Oct 2019 to Sep 2020. The other authors have no conflicts of interest to declare.

Ethical Statement: The authors are accountable for all aspects of the work in ensuring that questions related to the accuracy or integrity of any part of the work are appropriately investigated and resolved. Ethical approval was obtained from three general tertiary hospitals, including Xiangya Hospital, Hunan Provincial People's Hospital, and the First Affiliated Hospital of the University of South China. Written informed consent was obtained from all patients.

Open Access Statement: This is an Open Access article distributed in accordance with the Creative Commons Attribution-NonCommercial-NoDerivs 4.0 International
License (CC BY-NC-ND 4.0), which permits the noncommercial replication and distribution of the article with the strict proviso that no changes or edits are made and the original work is properly cited (including links to both the formal publication through the relevant DOI and the license). See: https://creativecommons.org/licenses/by-nc-nd/4.0/.

\section{References}

1. Gómez dos Santos VG, Burgos Revilla FJ, García González R. Glandular cystitis. Endovesical steroid treatment. Arch Esp Urol 2000;53:461-4.

2. Williamson SR, Lopez-Beltran A, Montironi R, et al. Glandular lesions of the urinary bladder:clinical significance and differential diagnosis. Histopathology 2011;58:811-34.

3. Xin Z, Zhao C, Huang T, et al. Intestinal metaplasia of the bladder in 89 patients: a study with emphasis on long-term outcome. BMC Urol 2016;16:24.

4. Mukhopadhyay S, Taylor W. Pathologic quiz case: bladder tumor in a 41-year-old man. Cystitis glandularis of intestinal type with mucin extravasation. Arch Pathol Lab Med 2004;128:e89-90.

5. Jankovic Velickovic L, Katic V, Hattori T, et al. Differences in the expression of mucins in various forms of cystitis glandularis. Pathol Res Pract 2007;203:653-8.

6. Sung MT, Lopez-Beltran A, Eble JN, et al. Divergent pathway of intestinal metaplasia and cystitis glandularis of the urinary bladder. Mod Pathol 2006;19:1395-401.

7. Liu X, Chen Z, Ye Z. Etiological study on cystitis glandularis caused by bacterial infection. J Huazhong Univ Sci Technolog Med Sci 2007;27:678-80.

8. Delnay KM, Stonehill WH, Goldman H, et al. Bladder histological changes associated with chronic indwelling urinary catheter. J Urol 1999;161:1106-8; discussion 1108-9.

9. Li A, Liu S, Lu H, et al. Clinical character of cystitis glandularis accompanied with upper urinary tract obstruction. Can Urol Assoc J 2013;7:E708-10.

10. Zhou X, Cui Y, Chen J, et al. UCA1 promotes cell viability, proliferation and migration potential through UCA1/miR204/CCND2 pathway in primary cystitis glandularis cells. Biomed Pharmacother 2019;114:108872.

11. Ma R, Li XB, Yang G, et al. [Feasibility of transurethral electro-resection as sole modality for the treatment of cystitis glandularis]. Zhonghua Yi Xue Za Zhi 2009;89:2571-3.

12. Lu Q, Jiang F, Xu R, et al. A pilot study on intravesical 
administration of curcumin for cystitis glandularis. Evid Based Complement Alternat Med 2013;2013:269745.

13. Luo M. Effect of transurethral resection combined with iodophor intravesical instillation for cystitis glandularis. Journal of Aerospace Medicine 2017;28:1222-3.

14. Chen L, Wu D, He H. Treatment and nursing of 393 female patients with glandular cystitis. Lingnan Modern Clinics in Surgery 2008;8:240-1.

15. Edwards PD, Hurm RA, Jaeschke WH. Conversion of cystitis glandularis to adenocarcinoma. J Urol 1972;108:568-70.

16. Thrasher JB, Rajan RR, Perez LM, et al. Cystitis glandularis. Transition to adenocarcinoma of the urinary bladder. N C Med J 1994;55:562-4.

17. Yi X, Lu H, Wu Y, et al. Cystitis glandularis: A

Cite this article as: $\mathrm{Hu} \mathrm{J}, \mathrm{Li} \mathrm{C}$, Guo X, Zhang H, Li H, Qiu D, Gong G, Liu P, Ren W, Chen J, Zu X. Development and validation of a predictive nomogram for the risk of recurrence in patients with cystitis glandularis. Ann Transl Med 2020;8(6):352. doi: 10.21037/atm.2020.02.102 controversial premalignant lesion. Oncol Lett 2014;8:1662-4.

18. Corica FA, Husmann DA, Churchill BM, et al. Intestinal metaplasia is not a strong risk factor for bladder cancer: study of 53 cases with long-term follow-up. Urology 1997;50:427-31.

19. Smith AK, Hansel DE, Jones JS. Role of cystitis cystica et glandularis and intestinal metaplasia in development of bladder carcinoma. Urology 2008;71:915-8.

20. Li Z, Ge G, Feng R, et al. Cyclooxygenase-2 and B-cell lymphoma-2 expression in cystitis glandularis and primary vesicle adenocarcinoma. BMC Urol 2014;14:2.

21. Gordetsky J, Epstein JI. Intestinal metaplasia of the bladder with dysplasia: a risk factor for carcinoma? Histopathology 2015;67:325-30. 